\title{
PENSIONES DE ALTOS FUNCIONARIOS EN LA JURISPRUDENCIA CONSTITUCIONAL: SOSTENIBILIDAD FINANCIERA*
}

\author{
Carlos Arturo Hernández Díaz** \\ Javier Francisco Franco Mongua***
}

Fecha de recibido: 26 de febrero de 2014

Fecha de aprobado: 29 de abril de 2014

Artículo resultado de Investigación

Forma de citación: Hernández, C. A. \& Franco, J. F. (2014). Pensiones de altos funcionarios en la jurisprudencia constitucional: sostenibilidad financiera. Revista Prolegómenos. Derechos y Valores, 17, 33, 77-96.

\begin{abstract}
Resumen
El régimen pensional de los altos funcionarios del Estado, considerado como especial, ha generado insostenibilidad financiera hasta antes del 2013. Tiempo en el que se adopta una visión más garantista de los derechos de igualdad, equidad, solidaridad y equilibrio financiero del sistema general de pensiones, típico de una posición del Análisis Económico del Derecho (AED). Entonces, el estudio de las decisiones jurisprudenciales respecto del sistema especial de pensiones de los altos funcionarios, en el marco de las herramientas económicas, es el claro ejemplo para decisiones posteriores que busquen la eficiencia jurídica y económica de los sistemas normativos.
\end{abstract}

\section{Palabras clave:}

Análisis Económico del Derecho, línea jurisprudencial, subreglas, eficiencia económica, ingreso base de liquidación (IBL) y abuso del derecho.

Artículo producto del proyecto de investigación titulado Activismo judicial en Colombia visto desde el análisis económico del derecho: el caso pensional. El proyecto se presentó y fue aprobado en la convocatoria número 19 del Sistema Universitario de Investigaciones (SUI) de la Fundación Universidad Autónoma de Colombia (FUAC). Los autores agradecen la colaboración en la búsqueda de jurisprudencia de Jaime Alberto Manrique Rodríguez.

** Abogado y especialista en derecho Administrativo de la Universidad Libre. Magister en Filosofía del Derecho. Candidato a Doctor en Derecho de la Universidad Externado de Colombia. Profesor Investigador de la Facultad de Derecho de la Fundación Universidad Autónoma de Colombia (FUAC). Correo electrónico carhedy@gmail.com

**** Investigador externo en calidad de contratista del Sistema Universitario de Investigaciones (SUI) de la Fundación Universidad Autónoma de Colombia (FUAC). Profesor de pregrado en las Facultades de Derecho de la Universidad Militar Nueva Granada (UMNG) y de la Universidad La Gran Colombia; además, docente de Postgrados en Jurisprudencia de la Universidad del Rosario. Abogado de la Universidad Libre y Sociólogo de la Universidad Nacional de Colombia. Magíster en Derecho Económico de la Universidad Externado de Colombia. Magíster en Derecho Comparado con énfasis en Derecho Económico de la Université de Paris 1 Panthèon Sorbonne y candidato al Doctor en Derecho de la Université de Paris Dauphine. Correo electrónico jfrank1980@gmail.com 


\title{
SENIOR GOVERNMENT PENSIONS IN THE CONSTITUTIONAL JURISPRUDENCE: FINANCIAL SUSTAINABILITY
}

\begin{abstract}
The retirement scheme for senior government officials, regarded as a special case, generated financial unsustainability before year 2013. In said year, a vision that guarantees equal rights, equity, solidarity and financial balance of the general pension system is adopted, a characteristic position of the Economic Analysis of Law (EAL). Therefore, the study of court decisions regarding the special retirement system for senior officials, in the context of economic tools, is a clear example for future decisions seeking legal and economic efficiency of regulatory systems.
\end{abstract}

\section{Keywords:}

Economic Analysis of Law, jurisprudential line, sub-rules, economic efficiency, base income (BI), law abuse

\section{PENSÕES SÊNIOR DO GOVERNO NA JURISPRUDÊNCIA CONSTITUCIONAL: A SUSTENTABILIDADE FINANCEIRA}

\begin{abstract}
Resumo
O regime de reforma por altos funcionários do governo, considerado como um caso especial, gerado insustentabilidade financeira antes do ano de 2013. No referido ano, uma visão que garanta direitos iguais, igualdade, solidariedade e equilíbrio financeiro do sistema de pensões em geral é adotada, uma posição característica de a análise econômica do direito (EAL). Portanto, o estudo de decisões judiciais a respeito do sistema de aposentadoria especial para altos funcionários, no contexto de instrumentos econômicos, é um exemplo claro para futuras decisões que buscam eficiência jurídica e económica dos sistemas de regulação.
\end{abstract}

\section{Palavras-Chave:}

Análise Econômica do Direito, Linha jurisprudencial, Sub-regras, Eficiência econômica, Renda base (BI), Abuso de direito.

\section{INTRODUCCIÓN}

La efectividad real o material de los derechos (fundamentales, sociales, económicos, culturales y colectivos) depende de los recursos financieros para poder realizarlos. Es decir, los derechos dependen de los recursos económicos existentes (Holmes \& Sunstein, 2011). En este sentido, el costo de los derechos es el debate que, de fondo, se ha generado en torno al régimen pensional especial de los altos funcionarios (congresistas, magistrados, entre otros), relacionado con la sostenibilidad financiera. 
La determinación de la sostenibilidad financiera mediante la responsabilidad fiscal es trascendente cuando se hace en uso de material normativo como normas jurídicas (constitucionales, reformas constitucionales o legales) o subreglas, definidas por la jurisprudencia de la corte constitucional. Es por esta razón que se hace necesario construir una línea jurisprudencial que permita destacar las sentencias importantes frente al tema y construir teorías estructurales respecto de la posición que ha tenido la Corte Constitucional en relación con el problema jurídico que se plantea.

La importancia de este estudio mediante la metodología descriptiva, explicativa y lógico deductiva es poder conocer el desarrollo que ha tenido la observancia del derecho desde el criterio transversal de la eficiencia económica (Posner, 2007). Criterio que ha venido siendo uno de los postulados primordiales del Análisis Económico del Derecho (AED) o Law and Economics. Esta corriente se constituye en una metodología de estudio del derecho, como objeto, en el que se aplican las herramientas económicas (de la teoría macroeconómica y economía del bienestar) al derecho y sus instituciones jurídicas (Cooter \& Ulen, 2002) régimen pensional.

Entonces, el marco en el que se desarrollará la presente investigación es el AED y, para ello, se tomarán conceptos previos sobre la construcción de líneas jurisprudenciales: escenarios constitucionales, patrón fáctico, tipos de sentencias y balance constitucional. Posteriormente, se planteará el problema jurídico que se pretende resolver; en él se abordarán las técnicas de investigación de una línea jurisprudencial: punto y sentencia arquimédica, ingeniería reversa y telaraña y puntos nodales de jurisprudencia. Finalmente, se hará una conclusión respecto al problema jurídico planteado y la sentencia dominante, en el marco del análisis económico del derecho (López, 2006).

\section{ELEMENTOS TEÓRICOS PREVIOS}

La línea jurisprudencial se entiende como una idea abstracta que determina la posible solución de un problema jurídico (López,2006) ${ }^{1}$. Es decir, el análisis dinámico de la jurisprudencia constitucional permite determinar una serie de líneas jurisprudenciales que la Corte Constitucional, desde el inicio de sus actividades como guardián y salvaguarda de la constitución (artículo $241 \mathrm{CP}$ ), ha determinado (López, 2006) $)^{2}$ para la solución de un problema jurídico bien definido y actual. Según la descripción que realiza el profesor Diego López Medina, la línea jurisprudencial permite identificar las subreglas que se encuentran vigentes en un momento determinado respecto a un patrón fáctico concreto, configurando teorías estructurales (López, 2006).

Al realizar la línea jurisprudencia mediante la graficación se puede establecer una serie de posibilidades, de acuerdo con la estabilidad de las decisiones coherentes entre sí: 1) líneas jurisprudenciales bien definidas; 2) líneas jurisprudenciales caóticas o polémicas; 3) líneas jurisprudenciales de cambio radical de forma incremental; 4) líneas jurisprudenciales de cambio radical de forma en el que existen momentos determinados y; 5) determinación de las decisiones colegiadas según las convicciones jurídicas, políticas, religiosas y, en general, ideológicas de los magistrados.

1 Para el presente escrito, se tomarán las categorías conceptuales definidas por el profesor Diego López Medina, en su texto el derecho de los Jueces. Entonces, al ser este un trabajo en el cual se suscriben los conceptos definidos por el mencionado texto se tomaran de forma estricta y se desarrollara el presente escrito conforme a ellos. Véase López, 2006.

2 De forma caótica, en la medida en que se presentan los casos concretos y estos son solucionados por la corte. Es por ello que el analista jurídico dentro del marco del análisis dinámico del precedente constitucional determina la coherencia lógica y la evolución de la jurisprudencia en el tiempo. Confr. López, 2006. 
Entonces, tomando la primera de estas gráficas, la línea jurisprudencial bien definida se puede concretar la denominada fuerza gravitacional del precedente que se presenta cuando dentro de una línea jurisprudencial bien definida un caso análogo fácticamente tendría que caer en el mismo sitio, relativamente. Es decir, la relatividad se expresa en razón de la sombra decisional (López, 2006) ${ }^{3}$ que aparece con los fallos que deberán encontrarse dentro de un rango considerable y razonable frente al fallo anterior.

La sombra decisional se considera como un espacio más o menos amplio en el cual los jueces deben seguir el precedente constitucional pero que por las técnicas de interpretación se incluye un elemento subjetivo e individual de quien las usa. Sin embargo, un elemento objetivo dentro de La línea jurisprudencial es la identificación de las clases de sentencias que pueden encontrarse. En este sentido, el profesor Diego López Medina clasifica las sentencia al interior de una línea jurisprudencial de la siguiente manera: 1) las sentencias importantes o sentencias hito y; 2) las sentencias no importantes.

Dentro de las primeras están: a) sentencia fundadora de línea, que se encuentra dentro del periodo inicial de la actividad ejercida por mandato constitucional de la Corte Constitucional (1991-1993) ${ }^{4}$; contienen relatos históricos y derecho comparado, plagadas de dictas y se identifican por no citar jurisprudencia constitucional anterior como precedente, esto es, se basan en el vacío jurisprudencial que permite la puesta en marcha de una posición inicial para fallar; b) sentencia consolidadora

3 La sobra decisional es un espacio más o menos amplio en el que el juez tiene movilidad para establecer su fallo de acuerdo con sus convicciones políticas y jurídicas expresadas mediante las técnicas de interpretación. Dicha movilidad está limitada en el acatamiento, sea horizontal o vertical, del precedente. (López,2006).

4 Frente a este periodo, debe decirse que parece impreciso,por cuanto la Corte Constitucional inició su actividad como salva guarda de la Constitución Política de 1991 en el año de 1992, para lo cual lo ideal sería el periodo de 1992 a 1994. de línea, se considera que es aquella en la que se realiza una definición por autoridad de la subregla, respetando las definiciones hechas por sentencias anteriores y decanta o filtra el balance constitucional hecho por la sentencia fundadora; c) sentencia modificadora de línea, esta es aquella que realiza una modificación jurisprudencial al introducir cambios importantes definidos por autoridad, no respetando precedentes; d) sentencia reconceptualizadora de línea, se da cuando revisa la línea jurisprudencial introduciendo nuevas teorías e incluyendo una redefinición de la ratio decidendi y; e) por último, al interior de la línea jurisprudencial se puede encontrar la sentencia dominante o principal, que a consideración del analista jurídico, es la que se encuentra vigente al momento de plantear el problema jurídico bien definido (López, 2006) ${ }^{5}$ y lo resuelve de forma concreta, comúnmente son sentencias recientes (López, 2006).

\section{PROBLEMA JURÍDICO BIEN DEFINIDO6: ENCABEZADO DE LA LÍNEA JURISPRUDENCIAL}

El problema jurídico, como lo define el Profesor Diego López, es la pregunta de investigación, como ruta de orientación para el analista jurídico y el encabezamiento de la línea jurisprudencia, de ahí la importancia de definirlo adecuadamente. En este sentido, los criterios objetivos y claros para definirlo bien son los siguientes: que este contenga un nivel medio de abstracción, que exista un patrón factico o escenarios constitucionales (aun cuando no puedan existir- definicionales-) y un concepto abstracto, sea un derecho o principio, que englobe o enmarque los escenarios constitucionales que de este puedan surgir, pero, sobre todo, que su formulación sea concreta.

5 Este concepto se acompasa con un nivel medio de abstracción en coherencia recíproca con el escenario constitucional definido; configurando el problema jurídico. Confr. López, 2006.

6 El problema jurídico puede ser definicional o de carácter propiamente factico, pero también puede estar relacionado con la parte dogmática o con la parte orgánica de la Constitución Política. Véase, López, 2006. 
Con todo se pueden identificar, en el análisis del texto precitado, que existen dos tipos de problemas jurídicos consistentes en quitar al investigador en su labor. Por un lado, el problema jurídico que contiene escenarios constitucionales bien definidos, esto es, concretos y específicos dentro de un contexto determinado atendiendo a elementos facticos. Y por el otro, un problema jurídico definicional, que plantea en un nivel medio de abstracción y es formulado concretamente para determinar definiciones jurisprudenciales, realizadas por la jurisprudencia constitucional y demás materiales normativos (constitución y leyes) a lo largo de la actividad de la corte en el control de constitucionalidad abstracto y concentrado o en su control concreto y difuso.

Bajo la premisa de estas definiciones previas, relativas al problema jurídico para la estructuración de una línea jurisprudencial, tenemos que para el presente escrito este es: ¿La aplicación actual del régimen pensional especial para congresistas, magistrados de las altas cortes y de otros funcionarios a los que es aplicable la normativa del artículo 17 de La ley número 4 de 1992, vulnera los principios de igualdad (arttículo $13 \mathrm{CP}$ ), universalidad, solidaridad y eficiencia (establecidos en el artículo 48 de la Carta Fundamental-reformado por el Acto Legislativo número 01 de 2005) en virtud: (i) de la inexistencia de un tope del valor pensional; (ii) del ajuste de la mesada pensional al aumento de salario mínimo legal vigente; (iii) en razón a que la liquidación pensional sea conforme a lo devengado por todo concepto en el último año y; (iv) la desproporción entre las cotizaciones y el valor final de la pensión adquirida en el marco de la sostenibilidad financiera del régimen pensional? El problema jurídico se plantea bajo la premisa de la existencia de un régimen pensional especial para altos funcionarios que ha tenido una interpretación distinta al fin del ordenamiento constitucional, vulnerando los derechos de otros cotizantes y beneficiarios del régimen pensional general. Es por ello que el problema jurídico definido intenta identificar si existe o no la vulneración de los principios de solidaridad, eficiencia, universalidad e igualdad, en el marco de la sostenibilidad fiscal.

De ser afirmativa la respuesta a lo largo de la jurisprudencia o por lo menos en la sentencia dominante, es necesario determinar cuáles son las vías o los factores por los cuales estas pensiones especiales de altos funcionarios vulneran los derechos de los más desprotegidos y pone en amenaza el sistema pensional, desde la perspectiva de la sostenibilidad fiscal. Entonces, como se evidencia, aparentemente, es un problema jurídico de carácter definicional por encontrarse en choque principios y derechos en el marco de un régimen pensional especial y general.

Sin embargo, esta apariencia se desvirtúa cuando se refleja la aplicación práctica de estos conceptos abstracto, en hechos como: (i) la afectación negativa al sistema financiero de sostenibilidad del régimen pensional; (ii) la vulneración del principio material de igualdad; (iii) abuso del derecho o fraude a la ley; (iv) restricción sostensible de la universalidad y solidaridad; (iv) la ineficiencia económica que por efecto multiplicador generara la imposibilidad física y financiera de proteger otros derechos de igual entidad o naturaleza y; (v) la homogenización del régimen pensional en cuanto se busca una acción afirmativa, en el marco de la igualdad material, por diferenciación económica, social o cultural de las personas que participan en este.

\section{TÉCNICAS DE INVESTIGACIÓN DE UNA LÍNEA JURISPRUDENCIAL}

El profesor Diego López propone unas técnicas de investigación para una línea jurisprudencial que, desde su experiencia $(2006)^{7}$, permitirán encontrar y determinar estructuralmente la

\footnotetext{
Es una propuesta que realiza el jurista como técnica que le ha permitido estructurar diferentes líneas jurisprudenciales, entre ellas, la relacionada con el problema jurídico de la fuerza vinculante de la jurisprudencia constitucional. Véase López, 2006.
} 
línea jurisprudencial propiamente dicha. Estas técnicas las describe como el punto arquimédico de apoyo, la ingeniería reversa y la telaraña y los puntos nodales de la jurisprudencia. Técnicas que serán usadas en el presente trabajo para poder estructurar la línea jurisprudencial, ya encabezada por el problema jurídico planteado.

\section{El Punto Arquimédico de Apoyo: Sentencia Arquimédica}

La sentencia Arquimédica es el punto de apoyo que cumple con características, como ser una sentencia lo más reciente posible, contener el mismo o similar escenario constitucional al planteado dentro del problema jurídico, $y$ tener citas jurisprudenciales en cantidad sobre el mismo factor factico, algunas veces. Entonces, para el caso concreto bajo estudio en este escrito, se tiene que la Sentencia Arquimédica que cumple con estos requisitos es la Sentencia C- 258 del 07 de mayo de 2013, MP. Jorge Ignacio Pretelt Chaljub, exp. núm. D-9173 y D-9183.

Esta sentencia es el resultado de la demanda de inconstitucionalidad contra el artículo 17 de la Ley 4 de 1992 referente a:

8 Demandantes: Germán Calderón España y Dionisio Enrique Araujo Angulo,Magistrado Ponente.
Artículo 17. El Gobierno Nacional establecerá un régimen de pensiones, reajustes y sustituciones de las mismas para los Representantes y Senadores. Aquéllas y éstas no podrán ser inferiores al $75 \%$ del ingreso mensual promedio que, durante el último año, y por todo concepto, perciba el Congresista. $Y$ se aumentarán en el mismo porcentaje en que se reajuste el salario mínimo legal. PARÁGRAFO. La liquidación de las pensiones, reajustes y sustituciones se hará teniendo en cuenta el último ingreso mensual promedio que por todo concepto devenguen los Representantes y Senadores en la fecha en que se decrete la jubilación, el reajuste, o la sustitución respectiva.

Por lo tanto, este es el punto de partida para realizar la línea jurisprudencial conforme al problema jurídico definido y a las técnicas de investigación, aquí ya reseñadas.

\section{Ingeniería Reversa: Nicho de Citaciones}

Al realizar la ingeniería inversa en la confección del nicho citacional es necesario referenciar todos los materiales normativos, como son: normas provenientes de la potestad legislativa, provenientes de la potestad reglamentaria y las decisiones judiciales precedentes citadas en la Sentencia Arquimédica. Este nicho citacional se realiza en un primer y segundo nivel. 


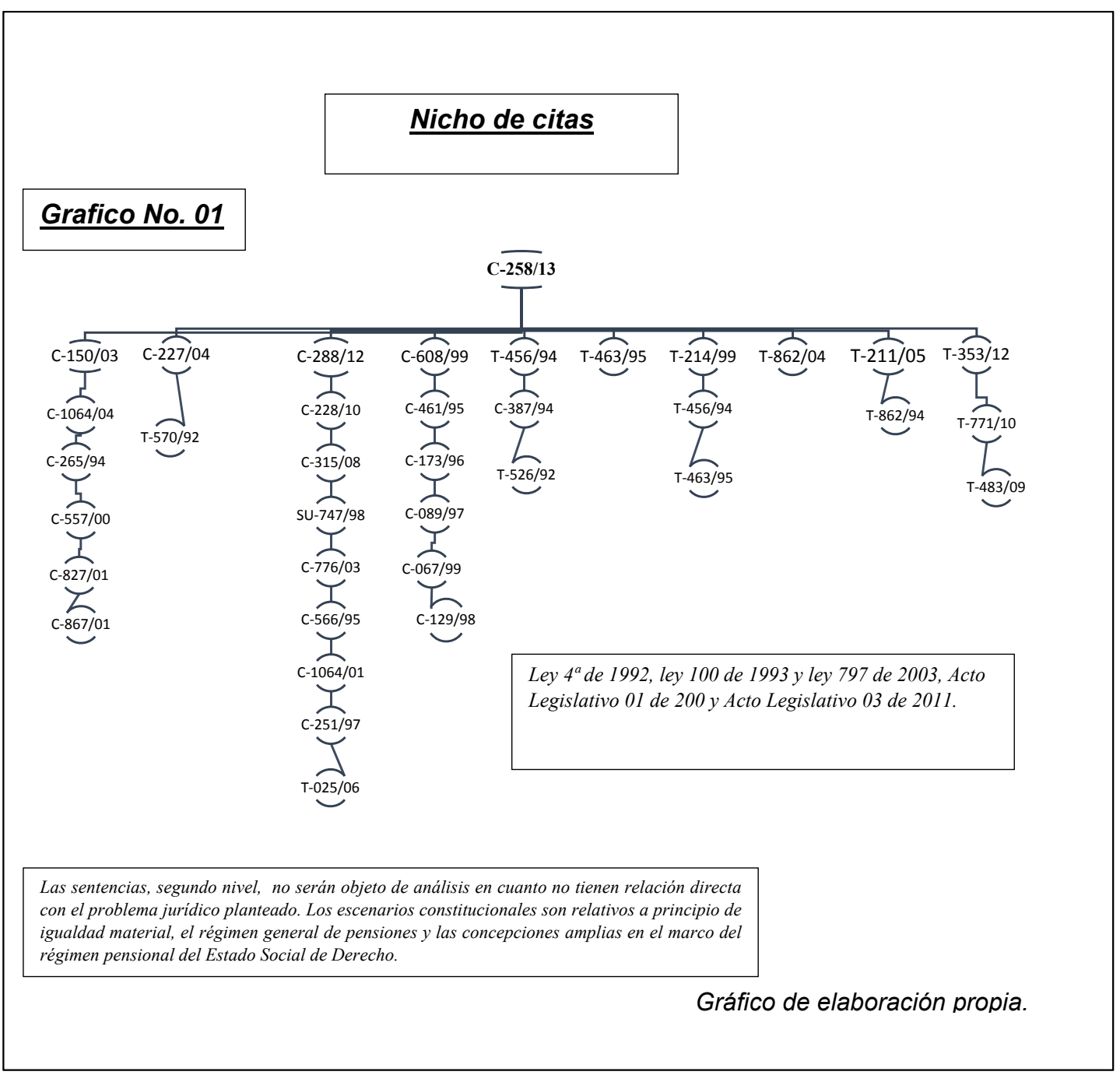

\section{Telaraña y Puntos Nodales de la Jurisprudencia}

Teniendo en cuenta que la Telaraña y los Puntos Nodales de Jurisprudencia se generan en el estudio del Nicho Citacional,en donde se encuentran ciertos puntos mencionados y referenciados en una cantidad de sentencias, que ordinariamente coinciden con las sentencias hito de la línea jurisprudencial, es necesario definir la clasificación de las sentencias al interior de una línea jurisprudencial, como conceptos previos que se verán aplicados en la clasificación de las sentencias pertenecientes a la línea jurisprudencial que se desarrolla. En este sentido, el profesor Diego López Medina clasifica las sentencias al interior de una línea jurisprudencial, de la siguiente manera: 1) las sentencias importantes o sentencia hito y; 2) las sentencias no importantes.

Dentro de las primeras están: a) sentencia fundadora de línea, que se encuentra dentro del periodo inicial de la actividad ejercida por 
mandato constitucional de la Corte Constitucional (1991-1993) ; contienen relatos históricos y derecho comparado, plagadas de dictas $y$ se identifican por no citar jurisprudencia constitucional anterior como precedente, esto es, se basan en el vacío jurisprudencial que permita la puesta en marcha de una posición inicial para fallar; b) sentencia consolidadora de línea, se considera que es aquella en la que se realza una definición por autoridad de la subregla, receta las definiciones hechas por sentencias anteriores y decanta o filtra el balance constitucional hecho por la sentencia fundadora; c) sentencia modificadora de línea, esta es aquella que realiza una modificación jurisprudencial al introducir cambios importantes definidos por autoridad; d) sentencia reconceptualizadora de línea, se da cuando revisa la línea jurisprudencial introduciendo nuevas teorías e incluyendo una redefinición de la ratio decidendi y; e) por último, al interior de la línea jurisprudencial se puede encontrar la sentencia dominante o principal, que a consideración del analista jurídico, es la que se encuentra vigente al momento de plantear el problema jurídico bien definido y lo resuelve de forma concreta. Comúnmente son sentencias recientes (López, 2006).

En este orden de ideas, la existencia de este régimen especial de pensiones fue reconocido constitucionalmente, mediante sentencias de control constitucional o control concreto (ejercido por la revisión de las sentencias resultado de una acción de tutela emitida por jueces de instancia), por su trascendencia e importancia: T-456 de 1994; T-463 de 1995; T- 214 de 1999; C-608 de 1999; T-862 de 2004; T- 211 de 2005, entre otras. En estas providencias se dijo que asistía el derecho a todos los ex congresistas para recibir su pensión de jubilación, conforme a lo indicado en el artículo 17 de la Ley 4. ${ }^{a}$ de 1992, esto es, para percibir una mesada pensional que no podía ser inferior al $75 \%$

9 Frente a este periodo, debe decirse que parece impreciso, por cuanto la Corte Constitucional inició su actividad como salva guarda de la Constitución Política de 1991 en el año de 1992, para lo cual lo ideal será el periodo de 1992 a 1994. del ingreso mensual promedio que durante el último año, y por todo concepto, perciban los congresistas en ejercicio.

Entonces, estos diversos fallos plasmaron subreglas que para ese momento eran aplicables, según la interpretación constitucional que se había otorgado al régimen especial pensional de los altos funcionarios: congresistas, magistrados, entre otros. Es decir, que según el artículo 17 de la Ley 4 de 1992, a la interpretación constitucional por teoría del derecho viviente se entiende bajo los siguientes criterios: (i) no solo son beneficiarios del régimen de transición los que al $1 .^{\circ}$ de abril de 1994 tienen la dignidad de magistrados o congresistas, sino aquellos que adquieran con posterioridad tal dignidad, siempre que se encuentren en el régimen de transición general del artículo 36 de la Ley 100 de 1993; (ii) el IBL aplicable es lo devengado, por todo concepto, durante el último año $y$ no los 10 años últimos, como lo consagraba el régimen general dispuesto en el artículo 36 inciso $3 .^{\circ}$ de la Ley 100 de 1993; (iii) la mesadas no se encuentran sometidas a un tope; (iv) el incremento anual de las mesadas pensionales se hace conforme al aumento del salario mínimo.

Sin embargo, dentro de las decisiones que favorecieron la sostenibilidad del régimen pensional, al ser eficiente y al tener proporción entre el aporte y el beneficio recibido, fue la sentencia C-608 de 1999. Sentencia de constitucionalidad que delimitó el alcance de los denominados factores de liquidación, en los cuales se debía considerar la liquidación conforme a los rubros que devengaran los congresistas como remuneración del servicio y lo específicamente recibido por cada beneficiario. Fallo este que en la práctica no fue aplicado plenamente por las autoridades judiciales, quienes siguieron tomando cualquier rubro recibido por el beneficiario: congresistas y magistrados.

Se concluye, entonces, que la normativa y la jurisprudencia han ordenado el respeto a la integralidad del régimen de los congresistas, especialmente, han señalado que aquellos 
tienen derecho a una pensión de jubilación no inferior al $75 \%$ del ingreso mensual promedio que durante el último año, y por todo concepto, devenguen los congresistas en ejercicio.

Por otro lado, teniendo en cuenta, como se ha mencionado, que el legislador ha permitido la extensión de los beneficios dados a los ongresistas a otros funcionarios (Corte Constitucional, Sentencia C-258 de 2013) ${ }^{10}$, como por ejemplo, a los magistrados de las altas cortes, estos también gozan de tal derecho en el momento de la liquidación de la pensión, en caso de que optaran por dicho régimen en caso de que les fuera más favorable.

La sentencia reconceptualizadora de línea es la C-150 de 2003, en sede de control abstracto de constitucionalidad; se realiza una reconceptualización sobre las posibilidades reales y efectivas de intervención estatal en los procesos económicos. Esto es, que al hablar de sostenibilidad fiscal, como lo hemos venido haciendo, se hace necesario que la Corte Constitucional defina de qué manera esta se puede llevar a cabo por parte de los agentes económicos estatales interventores. En otras palabras:

\section{(...) la intervención del Estado en la} economía puede ser de diferente tipo, sin que siempre pueda efectuarse una diferenciación clara entre las formas de intervención correspondientes. Así, por ejemplo, en la doctrina se habla de in-

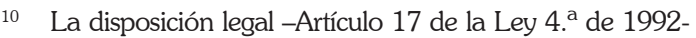
, «(...) prevista inicialmente para los Congresistas, es aplicable igualmente a otros servidores públicos en virtud de distintas normas; entre ellos se encuentran los Magistrados de Altas Cortes -artículo 28 del Decreto 104 de 1994- y ciertos funcionarios de la Rama Judicial, el Ministerio Público y órganos de control, como el Procurador General de la Nación -artículo 25 del Decreto 65 de 1998-, el Fiscal General de la Nación, el Registrador Nacional del Estado Civil, el Contralor General de la República, el Defensor del Pueblo, y los Delegados ante la Corte Suprema de Justicia y el Consejo de Estado -artículo 25 del Decreto 682 del 10 de abril de 2002». Corte Constitucional, Sentencia C- 258 de 2013. tervención estatal global, cuando versa sobre la economía como un todo, sectorial, cuando recae en una determinada área de actividad, o particular, si apunta a una cierta situación como por ejemplo a la de una empresa; de intervención estatal directa, cuando recae sobre la existencia o la actividad de los agentes económicos, o indirecta, cuando está orientada no a la actividad económica propiamente dicha sino al resultado de la misma; intervención unilateral, cuando el Estado autoriza, prohíbe o reglamenta una actividad económica, o intervención convencional, cuando el Estado pacta con los agentes económicos las políticas o programas que propenden por el interés general; intervención por vía directiva, cuando el Estado adopta medidas que orientan a los agentes económicos privados, o intervención por vía de gestión, cuando el Estado se hace cargo el (sic) mismo de actividades económicas por medio de personas jurídicas generalmente públicas(...) (Corte Constitucional, Sentencia C-150 de 2003).

Estas formas de intervención económica del Estado, decantadas por la doctrina y retomadas por la Corte Constitucional, muestran que son múltiples formas de intervención económica que realiza el Estado para poder orientar o direccionar los procesos económicos hacia el aumento del bienestar general. Sobre todo en la realización de los fines esenciales del Estado y en la protección y realización efectiva de los derechos fundamentales, y en procura de la realización efectiva, continua y progresiva de los derechos sociales, culturales, económicos y colectivos. Estas formas de intervención directa, indirecta, unilateral, colectiva son operaciones económicas realizadas por los agentes estatales económicos, pueden darse de manera simultánea o concomitante. Además de las mencionadas también se diferencian:

(...) de acuerdo con su función, la intervención del Estado en la economía 
también se puede agrupar en diferentes tipos. Algunos doctrinantes distinguen, entonces, tres clases de intervencionismo económico: conformativa, que establece los requisitos de existencia, formalización y funcionamiento de los actores económicos; finalística, que señala los objetivos generales o las metas concretas a los cuales han de propender los actores económicos; y condicionante, que propiamente fija las reglas de juego del mercado o de un sector económico. Adicionalmente, según su contenido, los actos de intervención estatal pueden someter a los actores económicos a un régimen de declaración -un nivel bajo de intervención que sólo exige que los actores económicos presenten a las autoridades determinada información-, un régimen de reglamentación, mediante el cual se fijan condiciones para la realización de una actividad, un régimen de autorización previa, que impide el inicio de la actividad económica privada sin que medie un acto de la autoridad pública que lo permita, un régimen de interdicción, que prohíbe ciertas actividades económicas juzgadas indeseables, o un régimen de monopolio, mediante el cual el Estado excluye del mercado determinadas actividades económicas, y se reserva para sí su desarrollo sea de manera directa o indirecta según lo que establezca la ley (Corte Constitucional, Sentencia C-150 de 2003).

Estas intervenciones económicas por parte del Estado se realizan en virtud del principio de sostenibilidad financiera, en ejercicio de la responsabilidad fiscal que le atiende a las entidades estatales de cualquier orden y nivel. Pero la responsabilidad fiscal, en virtud de la sostenibilidad financiera, no es la piedra de quiebre, restricción arbitraria o eliminación de los derechos fundamentales. Entonces, la Corte Constitucional en Sentencia C-227 de 2004 que fue resultado de una demanda de inconstitucionalidad instaurada contra el segundo inciso del parágrafo 4 del artículo 9 de la Ley 797 de 2003, en la cual se expresó que la sostenibilidad financiera no puede invocarse o argumentarse de manera genérica para limitar, restringir, prohibir o negar un beneficio generado de la seguridad social a personas pertenecientes a sectores vulnerables o en situación de debilidad manifiesta. Razón por la cual, declaró inexequible la expresión «menor de 18 años» del precepto acusado, en tanto excluía a las madres de las personas en condición de discapacidad que superaran ese rango de edad del beneficio de la pensión especial de vejez, a partir de razones de sostenibilidad financiera (Corte Constitucional, Sentencia C-227 de 2004), regla que también se afirma en la Sentencia C-258 de 2013. Por otro lado, la Corte Constitucional mediante Sentencia C-288 de 2012, manifestó que el criterio orientador de la sostenibilidad financiera mediante la responsabilidad fiscal no puede tomarse como un limitante de los derechos fundamentales sino, por el contrario, un criterio de realización progresiva de los derechos fundamentales (Corte Constitucional, Sentencia C-288 de 2012) ${ }^{11}$. En este orden de

11 La Corte manifestó: «Esta prohibición, en criterio de la Corte, debe leerse de manera armónica con los argumentos precedentes y, en especial, con los criterios fijados por la jurisprudencia constitucionalidad para determinar la iusfundamentalidad de una posición jurídica particular, explicados en los fundamentos anteriores. Es decir, cuando el Acto Legislativo determina que el SF debe ser compatible con la vigencia $y$ goce efectivo de los derechos fundamentales, quiere decir que la disciplina fiscal debe ceder ante la eficacia de esas posiciones jurídicas, conforme a la doctrina que sobre ese respecto ha fijado la Corte. Por ende debe rechazarse, por ser contraria a la Constitución, la interpretación según la cual la SF está basada en la distinción, ya superada, entre derechos de primera y segunda generación y que, además, el criterio de SF tiene por objeto aplazar o restringir el alcance de los derechos sociales, en oposición a los derechos fundamentales. Se ha señalado que esa restricción presenta profundos problemas dogmáticos y teóricos, de manera tal que la jurisprudencia constitucional ha concluido que la definición de un derecho como fundamental depende de factores específicos, relacionados con el vínculo entre la dignidad humana y la prestación correspondiente. Por ende, no de otra forma puede interpretarse la prohibición mencionada, 
ideas, la Corte Constitucional declaró que el Acto Legislativo 03 de 2011 no es una figura jurídica por reforma constitucional que se constituya en una sustitución de la Constitución en su concepto transversal del Estado Social de Derecho (Corte Constitucional, Sentencia C-288 de 2012).

Por otro lado, se destaca una sentencia, después de la C-258 de 2013 por su importancia, dentro del problema jurídico que se estudió, laT-353 de 2012, en la que la Corte Constitucional Sala Séptima de revisión- estableció:

Tal disposición lleva a replantear la forma como se han aplicado algunos regímenes, especialmente los que aún se encuentran vigentes en virtud del régimen de transición, y a cuestionar los factores que en reiteradas ocasiones se han tenido en cuenta para liquidar pensiones cuyos montos exceden el límite establecido por el constituyente. De modo que lo más sano, conveniente y razonable en aras de garantizar el principio sostenibilidad fiscal es la realización de una labor de revisión

sino de manera acorde con estos postulados, esto es, que cuando se hace referencia a los "derechos fundamentales", se entiende con claridad que son aquellas posiciones jurídicas que adquieren naturaleza iusfundamental, según la metodología antes expuesta. Esta explicación dista radicalmente de distinciones simplemente convencionales entre libertades $y$ derechos prestacionales, sino que versa sobre el grado de necesidad de protección constitucional en el caso concreto, valorado en términos de vigencia del principio de dignidad humana. || 70. Esta interpretación, a su vez, es consonante con otras previsiones del Acto Legislativo, como aquella que subordina al criterio orientador de la SF al logro de los fines del Estado Social y Democrático de Derecho. Si se parte de considerar que la cláusula del ESDD está basada, entre otros pilares, en el principio de dignidad humana y que, a su vez, este principio es el basamento teórico para la definición de una posición jurídica como iusfundamental, entonces la interpretación válida de la SF debe mostrarse compatible con la garantía de esas posiciones jurídicas, encuadradas en el concepto de "derechos fundamentales", que a su vez encuentra su definición concreta en la metodología explicada en este fundamento jurídico. Corte Constitucional, Sentencia C-288 de 2012. pensional, tanto de las pensiones que superan los 25 salarios mínimos legales mensuales vigentes concedidas antes del 31 de julio de 2010, como aquellas que se concedieron con posterioridad, para que a futuro el monto de dichas pensiones no supere el límite que el Constituyente determinó -es decir, 25 salarios mínimos legales mensuales vigentes-. (Corte Constitucional, Sentencia T-353 de 2012).

De esta sentencia se determina la siguiente subregla en materia económica, en especial de sostenibilidad financiera por intervención estatal del ordenamiento jurídico pensional: (i) que en los regímenes pensionales -de transición- no pueden ser desproporcionadas las mesadas pensionales, es decir, que el valor o monto de las mesadas pensionales deben ser ajustables bajo la sostenibilidad financiera del régimen pensional. Dejando además, como se evidencia en el texto citado literalmente, que el límite para este tipo de pensiones debe ser el de 25 salarios mínimos mensuales vigentes. Obiter dicta que es desarrollada por la Sentencia C-258 de 2013 dándole fuerza como una de las razones del fallo y definiéndola como subregla (ratio decidendi). Es decir, otorgándole fuerza vinculante por su conexidad con la decisium.

Con todo, las diferentes formas dadas por la doctrina y reconocidas por la jurisprudencia constitucional mencionada deben atender a criterios de eficiencia (Corte Constitucional, Sentencia C-258 de 2013) ${ }^{12}$ económica en la que se bus-

12 La Corte Constitucional « (...) ha definido la eficiencia como la elección de los medios más adecuados para el cumplimiento de los objetivos y la maximización del bienestar de las personas». Corte Constitucional, Sentencia C-258 de 2013. Concepto este que se complementa con la perspectiva dada por el análisis económico del derecho, que consiste en considerar que la eficiencia se encuentra al reducir costos y maximizar utilidades. Entonces, en un concepto homogéneo puede entenderse que la eficacia es la elección de los medios más adecuados que permitan reducir costos para el cumplimiento de los objetivos, en el cual se encuentra la maximización del bienestar o utilidad en general. Pareciere que esta definición dada 
quen los medios adecuados para reducir costos y maximizar la utilidad (económica, bienestar, interés común, entre otros) (Posner, 2007) ${ }^{13}$. Dicha eficiencia se logra cuando el Estado interviene en los procesos económicos porque se han presentado fallas en el mercado, mediante política económico-legal (Kirat \& Marty, 2012).

Respecto de la sentencia dominante -Sentencia C-258 de 2013- que a su vez es el punto arquimedico o sentencia arquimedica, se extraen explícitamente la siguientes subreglas en materia pensional y de sostenibilidad fiscal del régimen pensional, en virtud mayoritariamente de los postulados de la teoría del derecho viviente:

(i) (...) exequibilidad condicionada del artículo 17 de la Ley 4 de 1992 en el entendido que no puede extenderse el régimen pensional allí previsto, a quienes con anterioridad al $1^{\circ}$ de abril de 1994 , no se encontraban afiliados al mismo (...) (Corte Constitucional, Sentencia C-258 de 2013) ${ }^{14}$; (ii) (...) como

por la Corte Constitucional atendiera a una orientación conscuencialista propiamente representada por la Escuela de Chicago. Véase, Kirat \& Marty, 2012.

13 La utilidad se manifiesta en todos los aspectos de la vida, no solo económicos. Posner, 2007.

14 La expectativa legítima que han de tener los posibles beneficiarios de buena fe, sin abuso del derecho y/o fraude a la ley, pues dicha expectaiva debe atender a la teleología o fines del Estado social de derecho. En otras palabras, "[d]ado que la regla sobre beneficiarios, específicamente la posibilidad de que personas cobijadas por el régimen de transición que no estaban afiliadas al régimen especial del artículo 17 de la Ley 4 de 1992 el $1^{\circ}$ abril de 1994, se favorezcan de él, se desprende del derecho viviente y no tiene respaldo en expresión alguna del precepto acusado, en este caso la Sala considera que la fórmula por medio de la cual se debe retirar del ordenamiento tal contenido normativo, es la adopción de una sentencia interpretativa (...) Como ya la Sala explicó, las personas que no estaban afiliadas al régimen especial bajo estudio el $1^{\circ}$ de abril de 1994 -salvo la excepción prevista en el artículo 2 del Decreto 1293 de 1994, es decir, quienes ya habían sido congresistas antes del $1^{\circ}$ de abril de 1994- no tenían una expectativa legítima que generara una confianza merecedora de protección desde el punto de vista de la buena fe, y por tanto, no hay una razón factores de liquidación de la pensión, sólo podrán tomarse aquellos ingresos que hayan sido recibidos efectivamente por el beneficiario, que tengan carácter remunerativo del servicio y sobre los cuales se hubieren realizado las cotizaciones respectivas al sistema de pensiones(...) (Corte Constitucional, Sentencia C-258 de 2013); (iii) (...) en lo sucesivo, las mesadas pensionales de los beneficiarios del artículo demandado se ajustarán de conformidad con lo señalado en el sistema general de pensiones, es decir, con el IPC (...) (Corte Constitucional, Sentencia C-258 de 2013); (iv) (...) las mesadas correspondientes a pensiones reconocidas de conformidad con régimen especial bajo estudio, no podrán superar los 25 smmlv(...) (Corte Constitucional, Sentencia C-258 de 2013).

Frente a estas subreglas, que aparentemente desvirtuarían la teoría de los derechos adquiridos, es preciso mencionar que el artículo 58 de la Constitución y el Acto Legislativo 01 de 2005 que protegen aquellos derechos adquiridos, de manera limitada y no absoluta. Es decir, que estas normativas constitucionales no protegen los derechos adquiridos con fraude a la ley y abuso del derecho, es decir, no se entienden derechos adquiridos aquellos obtenidos con fraude a la ley o abuso del derecho. En otras palabras, «(...) no se configura propiamente un derecho adquirido cuando se ha accedido a éste (i) por medios ilegales, (ii) con fraude a la ley o (iii) con abuso del derecho. (... )» (Corte Constitucional, Sentencia C-258 de 2013) $)^{15}$.

que justifique un trato diferenciado preferencial». Corte Constitucional, Sentencia C-258 de 2013.

15 «En primer término, claro está, no constituirán derechos pensionales adquiridos aquellos que han sido causados a través de conductas como la alteración de documentos, la falsedad, entre otras. Este caso extremo puede haber ocurrido en muy pocas ocasiones, pero no por ello debe dejar de mencionarse. En segundo lugar, tal y como se explicó, en las figuras del fraude a la ley y abuso del derecho, se presenta un elemento objetivo que se traduce en el aprovechamiento de la 
Subreglas que atienden a la protección de los derechos de igualdad, universalidad, solidaridad y eficiencia. Principios que pueden verse desde dos perspectivas: 1) en sentido constitucional, en el cual son principios tendientes a garantizar los derechos de otras personas; igualdad entre las personas al interior del régimen, solidaridad que contribuye al sentido material de la igualdad cuando son quienes tienen más capacidad económica los que redistribuyen su riqueza en asignación a aquellos de menor capacidad económica. Universalidad, cuando se pretende mayor cobertura de contingencias y eficiencia, principio este propio de la sostenibilidad financiera del régimen pensional. 2) la segunda perspectiva engloba los otros principios en virtud de la eficiencia, como se mencionó, para la sostenibilidad financiera. Es decir, observar la solidaridad, equidad y universalidad dentro del análisis económico del derecho, en virtud de la eficiencia económica (maximizar utilidadbienestar social- y reducir costos -sostener financieramente el régimen pensional).

En este orden de ideas, la Sentencia C-258 de 2013 es clara al disponer otra subregla en materia de eficiencia y sostenibilidad financiera: (v) «(...) en el cumplimiento del presente fallo se han de adoptar las medidas conducentes a asegurar que el ahorro fiscal obtenido como resultado de los reajustes dispuestos en la parte resolutiva sean dirigidos al aumento de la cobertura del sistema general de pensiones (...)». Subregla en materia fiscal y de redistribución de los recursos que es favorable con los lineamientos del análisis económico del derecho en un marco de eficiencia económica y sostenibilidad fiscal del régimen de pensiones. Sin embargo, como lo menciona y justifica este fallo, el principio de sostenibilidad fiscal (Acto Legislativo 3 de 2011) no puede ser invocado para desconocer, vulnerar o eliminar derechos

interpretación judicial o administrativa de las normas o reglas, para fines o resultados incompatibles con el ordenamiento jurídico y aquél que invoca las normas de una forma claramente excesiva y desproporcionada que desvirtúa el objetivo jurídico que persigue». Corte Constitucional, Sentencia C-258 de 2013. fundamentales, como el de la seguridad social -pensión, por el contrario, los derechos no son de carácter absoluto y el interés general prima sobre el interés privado, es por ello que el artículo 48 reformado por el Acto Legislativo 01 de 2005 dispone «(...) garantizar la sostenibilidad financiera del sistema pensional en aras de asegurar su cobertura universal, la inclusión de las clases menos favorecidas y el pago efectivo de las mesadas pensionales» (Corte Constitucional, Sentencia C-258 de 2013).

En este sentido, dicho principio busca la maximización del bienestar mediante los medios adecuados, como la redistribución de los recursos. Dentro de estos medios adecuados se encuentran las herramientas jurídicas, entre ellas el Acto Legislativo número 01 de 2005 que da un reconocimiento al derecho a la seguridad pensional, como derecho fundamental que necesita de una sostenibilidad financiera continua y progresiva en realización del Estado Social de Derecho (Corte Constitucional, Sentencia C-258 de 2013) ${ }^{16}$. En otras reciente reformas constitucionales, sobre la sostenibilidad fiscal en procura de la efectivización de los derechos fundamentales, la Corte ha expresado:

\section{(...) las reglas de responsabilidad fiscal} $y$ el criterio de sostenibilidad tienen un carácter instrumental respecto de los fines $y$ principios del Estado Social de Derecho, en particular, son una herramienta útil para la realización progresiva de los contenidos prestacionales de las garantías constitucionales. Sin embargo, ha resaltado que la disciplina fiscal y la sostenibilidad financiera no pueden tomarse como fines

16 Con respecto a estas herramientas jurídicas se encuentran los principios fiscales en promoción y complemento del principio de sostenibilidad financiera. Véase, Corte Constitucional, Sentencia C- 258 de 2013, Acto Legislativo núm. 01 de 2005 y Acto Legislativo núm. 03 de 2011. «Parágrafo. Al interpretar el presente artículo, bajo ninguna circunstancia, autoridad alguna de naturaleza administrativa, legislativa o judicial, podrá invocar la sostenibilidad fiscal para menoscabar los derechos fundamentales, restringir su alcance o negar su protección efectiva». 
últimos del Estado ni justificar limitaciones de los derechos fundamentales (Corte Constitucional, Sentencia C-258 de 2013).

Pero esta efectivización de los derechos fundamentales y prestacionales son amenazados por la existencia de un régimen de transición que acoge el régimen pensional de altos funcionarios. Pues los factores de liquidación de la pensión, el ajuste que este monto tiene con base al salario mínimo mensual vigente y la desproporción entre lo cotizado y lo realmente establecido como valor pensional generan un agujero fiscal en el régimen general y universal de pensiones.

Finalmente, es importante destacar que la sostenibilidad financiera mediante la responsabilidad fiscal del Sistema General de Pensiones ha sido una preocupación continua $y$ transversal en la reforma constitucional, las leyes y la jurisprudencia constitucional actual que han enfatizado y favorecido la sostenibilidad financiera para la realización efectiva de los derechos fundamentales y sociales. Esta razón es la que asegure la eliminación de mesadas pensionales desproporcionadas. Pues se busca que las leyes y la jurisprudencia observen y orienten mediante la eficiencia económica la garantía efectiva de los derechos de las generaciones venideras.

Siendo un referente indirecto del problema jurídico planteado, es indispensable citar la sentencia del 26 de febrero del 2014 de la Sala Laboral de la Corte Suprema de Justicia que resuelve la impugnación de una sentencia, resultado de una acción de tutela interpuesta en la Sala Laboral del Tribunal Superior de Distrito Judicial de Bogotá D. C., contra el Fondo de Pensiones de Congresistas por haber reducido de forma automática el monto pensional de la cónyuge supérstite (pensión de sobreviviente) de un exrepresentante a la cámara, fundamentando, a su parecer, dicho proceder en la orden impartida por la Sentencia C-258 de 2013 de la Corte Constitucional (Corte Suprema de Justicia. 2014).

Entonces, las autoridades encargadas del pago, modificación, reducción o revocatoria de las pensiones adquiridas, conforme al artículo 17 de la ley 4. ${ }^{\text {a }}$ de 1992 y reguladas, caso por caso, por la Sentencia C-258 de 2013, deben agotar el debido proceso, esto es, mediante acto administrativo y/o decisión judicial motivar la reducción del moto pensional adquirido. En este sentido, debe especificar a cuál de los casos previstos en la Sentencia C-258 de 2013 se circunscribe el caso concreto, los motivos por los cuales se pretende la reducción del monto pensional, en el marco de los 25 SMMLV, y agotar todos los procedimientos administrativos que tiene el beneficiario de la pensionada para oponerse, en ejercicio del derecho de defensa, a la decisión adoptada.

Por tanto, el amparo del derecho al debido proceso (artículo $26 \mathrm{CN}$ ) es predominante al entenderse que el ajuste no es automático con fundamento en orden jurisprudencial impartida. Se concluye, frente al problema jurídico planteado, que la sostenibilidad financiera del sistema especial pensional no puede desconocer las garantías procesales de aquellos que han adquirido un derecho pensional. Se busca la sostenibilidad financiera sin desconocimiento de las garantías del debido proceso, atendiendo a la confianza legítima y la buena fe de los ciudadanos titulares de derechos de la seguridad social (pensión de sobreviviente). (Corte Suprema de Justicia, 2014).

\section{BALANCE CONSTITUCIONAL: GRÁFICA DE LÍNEA JURISPRUDENCIAL}

En el balance constitucional, tomando como referente el problema jurídico planteado, se ubican respuestas opuestas y dentro de este rango o espacio se determinan las posiciones jurídicas evocadas mediante las decisiones judiciales constitucionales (López,2006). En concreto, se busca definir la posición jurídica, mediante jurisprudencia constitucional, respecto de la sostenibilidad financiera del Sistema General de Pensiones, eniendo en cuenta los cuatro factores enunciados en el problema jurídico planteado relacionado con el régimen pensional de altos funcionarios. 


\section{Gráfico 2.}

\begin{tabular}{|c|c|c|}
\hline & $\begin{array}{l}\text { En materia de sostenibilidad financiera: ¿la apli- } \\
\text { cación actual del régimen pensional especial para } \\
\text { congresistas, magistrados de las altas cortes y de otros } \\
\text { funcionarios, a los que es aplicable la normativa del } \\
\text { artículo } 17 \text { de la Ley número } 4 \text { de } 1992 \text {, vulnera los } \\
\text { principios de igualdad (artículo } 13 \text { CP), universalidad, } \\
\text { solidaridad y eficiencia (artículo } 48 \text { Fundamental } \\
\text { reformado por el AL número } 01 \text { de } 2005 \text { ) en razón del } \\
\text { principio de sostenibilidad financiera? }\end{array}$ & \\
\hline $\begin{array}{l}\text { Es vulnerado el } \\
\text { principio de sosteni- } \\
\text { bilidad financiera por } \\
\text { reconocimiento de } \\
\text { pensiones sin tope, } \\
\text { ajustadas al SMMLV, } \\
\text { liquidadas con base } \\
\text { en lo devengado } \\
\text { por todo concepto } \\
\text { en el último año y } \\
\text { desproporcionadas en } \\
\text { cuanto a cotización- } \\
\text { valor de pensión, en } \\
\text { el marco del régimen } \\
\text { especial para altos } \\
\text { funcionarios. }\end{array}$ & $\begin{array}{l}\text { C-150/03 } \\
\text { C-227/04 } \\
\text { Act. Leg. 01/05 } \\
\text { Act. Leg. 03/11 } \\
\text { C-288/2012 } \\
\text { T-353/12 } \\
\text { C-258/13 } \\
\text { S. } 26 / \text { febr./ } 2014\end{array}$ & $\begin{array}{l}\text { No se vulnera el principio } \\
\text { de sostenibilidad financie- } \\
\text { ra al ser acorde a derecho } \\
\text { el reconocimiento de pen- } \\
\text { siones sin tope, ajustadas } \\
\text { al SMMLV, liquidadas con } \\
\text { base a lo devengado por } \\
\text { todo concepto en el último } \\
\text { año y estimando positiva } \\
\text { una relación cotización- } \\
\text { valor de pensión, en el } \\
\text { marco diferenciador y } \\
\text { de derechos adquiridos } \\
\text { del régimen especial } \\
\text { para altos funcionarios y } \\
\text { régimen pensional general. }\end{array}$ \\
\hline
\end{tabular}

Fuente: Gráfico elaboración propia

CONCLUSIÓN: Law and Economics y artículo 17 de la Ley 4 de 1994

El Sistema pensional en Colombia, desde su creación, ha tenido inestabilidad financiera y fiscal, sobre todo, aquellos regímenes especiales para los servidores públicos y de elección popular (senadores y representantes a la cámara). La Corte Constitucional ha contribuido, mediante sus decisiones en sede de control concreto (Decreto 2067 \& 2591 de 1991) ${ }^{17}$, desde su visión populista a la garantización de los derechos derivados de la seguridad social, en desmedro de la

17 La corte Constitucional en su sala de Selección ejerce la potestad de llevar a revisión las sentencias de tutela relevantes para el establecimiento de precedente constitucional. Decreto núm. 2067 de 1991 y Decreto núm. 2591 de 1991. sostenibilidad fiscal. Sin embargo, la reciente posición de la Corte Constitucional respecto del régimen especial pensional de los congresistas y otros servidores públicos (Ley núm. 4 de 1992) restringe el abuso del derecho por parte de estos en beneficio, aparentemente indirecto, del sistema de financiación pensional especial. En el presente artículo, se determina la posición de la Corte Constitucional para continuar con unas conclusiones que contendrán una posición jurídica establecida.

La Corte Constitucional, recientemente, mediante Sentencia C-258 de 2013, - control abstracto- en revisión del artículo 17 de la Ley núm. 4 de 1992, ha considerado que existen 
restricciones respecto de la disposición legal de creación de un régimen especial, por parte del Gobierno Nacional, para los congresistas, magistrados y otros servidores públicos Es así como resolvió lo siguiente ${ }^{18}$ :

(i) son inexequibles las expresiones: (...) «durante el último año y por todo concepto», $y$ "se aumentarán en el mismo porcentaje en que se reajuste el salario mínimo legal», contenidas en el primer inciso del artículo 17 de la Ley 4 de 1992; también la expresión "por todo concepto», contenida en su parágrafo (...) (Corte Constitucional, Sentencia C-258 de 2013); (ii) son exequibles las demás expresiones del artículo mencionado, en el entendido de que (...) A) No puede extenderse el régimen pensional allí previsto, a quienes con anterioridad al $1 .^{\circ}$ de abril de 1994 , no se encontraren afiliados al mismo. B) Como factores de liquidación de la pensión solo podrán tomarse aquellos ingresos que hayan sido recibidos efectivamente por el beneficiario, tengan carácter remunerativo del servicio $y$ sobre los cuales se hubieren realizado las cotizaciones respectivas. C) Las reglas sobre ingreso base de liquidación (IBL) aplicables a todos los beneficiarios de este régimen especial, son las contenidas en los artículos 21 y 36, inciso tercero, de la Ley 100 de 1993, según el caso. D) Las mesadas correspondientes a pensiones reconocidas de conformidad con este régimen especial, no podrán superar los veinticinco (25) salarios mínimos legales mensuales vigentes, a partir del $1^{\circ}$ de julio de 2013; (iv) Las pensiones reconocidas al amparo del artículo 17 de la Ley 4 de 1992, con abuso del derecho o con fraude a la ley, en los términos del acápite de conclusiones de esta sentencia, se

18 Dentro del resuelve se encuentra la improcedencia de la solicitud de nulidad. revisarán por los representantes legales de las instituciones de seguridad social competentes, quienes podrán revocarlas o reliquidarlas, según corresponda, a más tardar el 31 de diciembre de 2013 y; (v) En los demás casos de pensiones reconocidas de manera contraria a lo dispuesto en los numerales i, ii y iii del ordinal tercero, quienes tienen a su cargo el reconocimiento y pago de las pensiones decretadas al amparo del artículo 17 de la Ley 4 de 1992 deberán en el marco de su competencia tomar las medidas encaminadas para hacer efectivo el presente fallo, aplicando en lo pertinente, los artículos 19 y 20 la Ley 797 de 2003, en los términos del apartado de conclusiones de esta sentencia (Corte Constitucional, Sentencia C-258 de 2013).

Los congresistas y magistrados, dentro del régimen especial o excepcional abolido por el Acto Legislativo 01 de 2005 y estando bajo el régimen de transición del artículo 36 de la Ley núm 100 de 1993, tomaban una posición de abuso del derecho ${ }^{19}$ y fraude a la ley ${ }^{20}$ que permitía que obtuvieran una posición económica- pensionalventajosa en desmedro de los derechos ajenos y el quebranto del ordenamiento jurídico, pues son principios irradiantes del Sistema de Seguridad Social la universalidad, la solidaridad, la sostenibilidad, eficiencia y la equidad.

Es decir, que desde el 2005, con la reforma al artículo 48 Fundamental, le son aplicables, sin discriminación, las condiciones del Sistema General de Pensiones a todos los residentes en Colombia, entre estos criterios se encuentra el tope máximo de liquidación y asignación de pensión en 25SMLMV y el tiempo y condiciones

19 En el cual es aplicable la norma jurídica pero en desmedro de su objetivo y en violación de derechos ajenos por el ejercicio del derecho propio.

20 Cuando se usan las opciones hermenéuticas de la disposición legal, no compatibles con los fines reales del ordenamiento jurídico. 
de salario en el que se debe calcular el Ingreso Base de Liquidación (IBL). Entonces, este tipo de pensiones mayores, respecto de los topes máximos y mínimos o incluso sustrayéndose de estos, en cuanto a la posibilidad de liquidarla con un IBL mayor que el general, como lo afirma la Corte, ha generado un desmedro a los principios del Sistema General de Pensiones, por establecer los regímenes especiales para congresistas y magistrados ${ }^{21}$.

Dentro de estos principios, como se mencionó, encontramos la universalidad, la sostenibilidad, la equidad, la solidaridad y, especialmente, la eficiencia. Es decir, el principio de eficiencia económica, desde el Law and Economics ${ }^{22}$, es aquel que permite maximizar utilidad (en todos los aspectos de la vida, no solo los económicos) y reducir los costos (Posner, 2007). Esto es, la relación entre económica y derecho se evidencia cuando las herramientas económicas de análisis son aplicadas a las normas jurídicas (Posner, 2007). En este caso se evidencia que lo que la Corte Constitucional, desde el AED, decide es la eficiencia económica del Sistema General de Pensiones, toda vez que busca reducir los costos a pagar en pensiones y maximizar la utilidad económica, de bienestar y de efectividad de derechos para los demás residentes del país. Aun cuando existe una redistribución de la riqueza con este tipo de decisiones (Nuñez,2005), esta redistribución se justifica por el principio de solidaridad y maximización de utilidad universal de aquellos que tienen una posición económica desventajosa.

En otras palabras, el análisis económico del Derecho, desde sus escuelas Chicago, New Heaven y Neoinsitucionalismo, ha tratado y desarrollado el concepto de eficiencia económica y utilidad (este último desde Jeremy Bentham y Cessare Becaria- bajo la bandera

21 Un ejemplo de esto es el denominado Carrusel de pensiones de altos funcionarios.

22 Law and Economics o Análisis Económico del Derecho (AED). del utilitarismo). Este principio de eficiencia económica, aplicable a todo el ordenamiento jurídico, describe la posibilidad de que el derecho y, específicamente, las normas jurídicas contribuyan de forma eficaz al crecimiento o decrecimiento económico. En este sentido, se busca que las normas jurídicas creen una maximización de utilidad y una minimización de costos que beneficie, de ordinario, al interés general o común (Kirat \& Marty, 2012).

En el caso concreto, las normas que establecen la posibilidad de que el Gobierno Nacional cree un sistema especial de pensiones para congresistas y magistrados en una norma que al ser aplicable, y por su ambigüedad, vacíos y textura abstracta (artículo 17 de la Ley núm. 4 de 1992), genere posibles abusos del poder $y$, sobre todo, fraude a la ley. Pues la posibilidad de múltiples interpretaciones y el ejercicio del derecho de forma legítima es llevado a una concepción de elección racional (Posner, 2007) del individuo beneficiario.

La elección racional es un concepto económico en el que se parte del presupuesto de que todos los individuos racionales tomamos elección buscando siempre la mayor utilidad, para este caso económica- y la reducción al máximo de los costos. En este sentido, los magistrados y congresistas son individuos que toman decisiones racionales, en búsqueda de la maximización de sus utilidad respecto del mayor monto o valor de una pensión y la reducción de costos, como son disminuir o evitar los requisitos que son más rigurosos para los demás miembros del Sistema General de Pensiones en la obtención de la pensión correspondiente.

Sin embargo, la Corte Constitucional, en su fallo, restringe esa posibilidad de elección racional ejercida por magistrados y congresistas mediante el fraude a la ley y el abuso del derecho, al determinar (C-258 de 2013) que se busca el interés general, esto es, desde la utilidad común, reducir los costos generados por el pago de valores de altas pensiones a magistrados y congresistas que, en compara- 
ción con otros, tienen una muy favorable capacidad económica. Y aumentar la utilidad o maximizarla en cuanto se busca que aquellos menos favorecidos económicamente puedan verse afectados positivamente por la solidaridad e igualdad material que son los fundantes de un sistema pensional general o universal sostenible y equitativo.

En conclusión, la restricción que realiza en principio el acto Legislativo núm. 01 de 2005 y la jurisprudencia constitucional, en especial la Sentencia C- 258 de 2013, se justifica, ya que los magistrados y congresistas, tal vez no todos, adquirieron pensiones sustraídas en su valor de los máximos y mínimos, y bajo un IBL superior por las condiciones discriminadas de calcularlo. Todo esto bajo un abuso del derecho y fraude a la ley, que quebró gravemente los principios de sostenibilidad, equidad, solidaridad, universalidad y eficiencia que son el faro del Sistema General de Pensiones. Principio de eficiencia económica que fue garantizado, hasta ahora, mediante una decisión de constitucionalidad que, aunque genere una redistribución de la riqueza, desde el análisis económico del Derecho y desde los principios y derechos fundamentales de la Constitución de 1991, es justificable en beneficio de la mayor utilidad (población sin o de menor capacidad económica).

\section{REFERENCIAS}

Camargo, M. (2010). Aproximaciones al análisis económico del derecho. Bogotá D. C.: Universidad Externado de Colombia.

Cooter, \& Ulen. (2002). Derecho y Economia. México D. F.: Fondo de Cultura Económica.

Corte Constitucional. SentenciaT-526 de 1992. (M.P. Ciro Angarita Barón; Septiembre 18 de 1992).

Corte Constitucional. Sentencia T-570 de 1992. (M.P. Jaime Sanín Greiffenstein; Octubre 26 de 1992).

Corte Constitucional. Sentencia C-265 de 1994. (M.P. Alejandro Martínez Caballero; junio 2 de 1994).
Corte Constitucional. SentenciaC-387 de 1994. (M.P. Carlos Gaviria Díaz; Septiembre 1 de 1994).

Corte Constitucional. Sentencia T-456 de 1994. (M.P. Alejandro Martínez Caballero; Octubre 21 de 1994).

Corte Constitucional. SentenciaT-862 de 1994. (M.P. Ciro Angarita Barón; Septiembre 18 de 1994).

Corte Constitucional. Sentencia T-463 de 1995. (M.P. José Gregorio Hernández Galindo; Septiembre 20 de 1995).

Corte Constitucional. Sentencia C-566 de 1995. (M.P. Eduardo Cifuentes Muñoz; noviembre 30 de 1995).

Corte Constitucional. Sentencia C-251de 1997. (M.P. Alejandro Martínez Caballero; Mayo 28 de 1997).

Corte Constitucional. Sentencia SU-747 de 1998. (M.P. Eduardo Cifuentes Muñoz; Diciembre 2 de 1998).

Corte Constitucional. Sentencia T- 214 de 1999. (M.P. Vladimiro Naranjo Mesa; Abril 13 de 1999).

Corte Constitucional. Sentencia C-608 de 1999. (M.P. José Gregorio Hernández Galindo; Agosto 23 de 1999).

Corte Constitucional. Sentencia C- 557de 2000. (M.P. Vladimiro Naranjo Mesa; Mayo 16 de 2000).

Corte Constitucional. Sentencia C-827 de 2001. (M.P. Álvaro Tafur Galvis; Agosto 8 de 2001).

Corte Constitucional. Sentencia C-867 de 2001. (M.P. Manuel José Cepeda Espinosa, Agosto 15 de 2001).

Corte Constitucional. Sentencia C-1064 de 2001. (M.P. Manuel José Cepeda Espinosa; Octubre 10 de 2001).

Corte Constitucional. SentenciaC-150 de 2003. (M.P. Manuel José Cepeda Espinosa; Febrero 25 de 2003). 
Corte Constitucional. Sentencia C-776 de 2003. (M.P. Manuel José Cepeda Espinosa; Septiembre 9 de 2003).

Corte Constitucional. Sentencia C-227 de 2004. (M.P. Manuel José Cepeda Espinosa; Marzo 8 de 2004).

Corte Constitucional. Sentencia T-862 de 2004. (M.P. Marco Gerardo Monroy Cabra; Septiembre 3 de 2004).

Corte Constitucional. Sentencia C-1064 de 2004. (M.P. Manuel José Cepeda Espinosa; Octubre 30 de 2004).

Corte Constitucional. Sentencia T-211 de 2005. (M.P. Alvaro Tafur Galvis; Marzo 10 de 2005).

Corte Constitucional. Sentencia T-025 de 2006. (M.P. Manuel José Cepeda Espinosa; Agosto 11 de 2006).

Corte Constitucional. Sentencia C-315 de 2008. (M.P. Jaime Córdoba Triviño; Abril 9 de 2008).

Corte Constitucional. Sentencia T-483 de 2009. (M.P. Humberto Antonio Sierra Porto; Julio 21 de 2009).

Corte Constitucional. Sentencia C-228 de 2010. (M.P. Luis Ernesto Vargas Silva; Marzo 24 de 2010).

Corte Constitucional. Sentencia T- 711 de 2010. (M.P. Jorge Iván Palacio Palacio; Septiembre 8 de 2010).

Corte Constitucional. Sentencia C-288 de 2012. (M.P. Luis Ernesto Vargas Silva; Abril18 de 2012).

Corte Constitucional. Sentencia T-353 de 2012. (M.P. Jorge Ignacio Pretelt Chaljub; Mayo 15 de 2012).

Corte Constitucional. Sentencia C-258 de 2013. (M.P. Ciro Angarita Barón; Septiembre 18 de 2013).
Corte Suprema de Justicia- Sala Laboral. Sentencia 2584 de 2014 (M.P. Elsy del Pilar Cuello Calderón; Febrero 26 de 2014).

Decreto 104 de 1994. Por el cual se dictan unas disposiciones en materia salarial y prestacional de la Rama Judicial, del Ministerio Público, de la Justicia Penal Militar y se dictan otras disposiciones. Enero 3 de 1994.

Decreto 65 de 1998. Por el cual se dictan unas disposiciones en materia salarial y prestacional de la Rama Judicial, del Ministerio Público, de la Justicia Penal Militar y se dictan otras disposiciones. Enero 10 de 1998.

Decreto 682 de 2002. Por el cual se dictan unas disposiciones en materia salarial y prestacional de la Rama Judicial, del Ministerio Público, de la Justicia Penal Militar y se dictan otras disposiciones. Abril 10 de 2002.

Decreto 2067 de 1991. Por el cual se dicta el régimen procedimental de los juicios y actuaciones que deban surtirse ante la Corte Constitucional. Septiembre 4 de 1991

Decreto 2591 de 1991. Por el cual se reglamente la acción de tutela consagrada en el artículo 86 de la Constitución Política. Noviembre 19 de 1991.

Franco, J.F. (2011). Deontologismoy Consecuencialismo: Visiones y actuaciones de economistas y juristas. Bogotá D. C.: Universidad Libre.

Gonzáles, N. \& Crist, I. (2008). Estudios de Derechoy Economía. Argentina: Heliasta.

Holmes, S. \&. Sunstein, C. (2011). El Costo de los derechos;Por qué la libertad depende de los impuestos. The Cost of the Rights; Why the liberty depends on Taxes. (S. Mastrangelo, Trad.). Buenos Aires, Argentina: Siglo Veintiuno.

Kirat, T. (1999). Economie du droit, La Découverte, Collection Repères. Francia- París. Kirat, T., \& Marty, F. (2012). Economía del Derecho y de la regulación. 
economie du droit et de la réglementation. Bogotá D. C., Colombia: Universidad Libre.

Ley $4^{\mathrm{a}}$ de 1992. Mediante la cual se señalan las normas, objetivos y criterios que debe observar el Gobierno Nacional para la fijación del régimen salarial y prestacional de los empleados públicos, de los miembros del Congreso Nacional y de la Fuerza Pública y para la fijación de las prestaciones sociales de los Trabajadores Oficiales y se dictan otras disposiciones, de conformidad con lo establecido en el artículo 150, numeral 19, literales e) y f) de la Constitución Política Mayo 18 de 1992.

López, D. (2006). El derecho de los jueces. Bogotá D. C.: Universidad de los Andes.

Malloy, R (2000). Law and Market Economy, Cambridge: Cambridge University Press.

Mercuro,N \& Medema, S . (1997). Economics and the Law: from Posner toPost-modernism. Princeton: Princeton University Press.
Núñez, A. J. (2000). Antecedentes y principios fundamentales del análisis económico de la ley. En Colección de derecho económico. Homenaje a Enrique Low Murtra. Bogotá D. C.: Universidad Externado de Colombia.

Núñez, A. J. (2005). Manifiesto por una justicia constitucional responsable. Bogotá D. C.: Legis.

Stringham, E. (2001). "Kaldor-Hicks Efficiency and the Problem of Central Planning", pp. 41-50. En TheQuarterly Journal of Austrian Economics vol. 4, no. 2.

Pirou, G. (1939) Introducción al estudio de la economía política. Paris: Sirey.

Posner, R. (2007). Análisis Económico del Derecho. 2a edición. Trad. de Eduardo L. Suarez. México D. F.: Fondo de Cultura Económica (FCE).

Stringham, Edwar. (2001). Kaldor-Hicks Efficiency and the Problem of Central planning, the quarterly journal of Austrian economics. 